\title{
RANDOMISED TRIAL OF TOPICAL VERSUS SUB-TENON'S LOCAL ANAESTHESIA FOR SMALL-INCISION CATARACT SURGERY
}

\author{
T. D. MANNERS and R. L. BURTON \\ Norwich
}

\begin{abstract}
SUMMARY
Fifty unselected patients undergoing routine cataract surgery were randomised to receive either topical or sub-Tenon's local anaesthesia with $2 \%$ prilocaine administered by a blunt cannula. Visual analogue scales were used to assess pain during administration of subTenon's anaesthetic and pain during surgery, and any complications were noted. Sub-Tenon's anaesthesia proved to be entirely comfortable to administer, and allowed for a statistically significantly more pain-free operation, at the expense of some residual eye movement and an inevitable subconjunctival haemorrhage. Both techniques compared well with other studies assessing periorbital or retro-orbital injections, and both have significant safety advantages which are discussed in the context of the joint Royal Colleges report on ophthalmic anaesthesia. It is suggested that a combination of one or other technique could safely cover all requirements for intraocular surgery under local anaesthesia.
\end{abstract}

Local anaesthesia, combined with day case surgery and phacoemulsification, is becoming the treatment of choice for cataract surgery in the UK National Health Service. Recent reports have highlighted the risks of traditional methods of administration involving placement of sharp needles into the retro- or periorbital spaces,${ }^{1-6}$ and have led to the publication in the United Kingdom of a joint Royal Colleges report advising perioperative monitoring by an anaesthetist. ${ }^{3}$ Peribulbar administration may be safer, and is the route preferred by most anaesthetists giving blocks, but serious side effects have been reported, including globe perforation., ${ }^{7,8}$

Topical $^{9-12}$ and sub-Tenon's ${ }^{13,14}$ methods of administration are two options recently reported to give satisfactory operating conditions. Neither

Correspondence to: Mr T. D. Manners, FRCOphth, Ophthalmology Department, West Norwich Hospital, Bowthorpe Road, Norwich NR2 3TU, UK. involves blind placement of sharp needles into the orbit, and side effects would not be expected to include globe perforation, optic nerve penetration, compromise of the central nervous system, orbital haematoma or intravenous injection.

Topical anaesthesia has been shown to give satisfactory patient comfort during short intraocular surgery, and allows early visual rehabilitation with an excellent cosmetic appearance. Some surgeons are, however, concerned by the full eye movements, which require a firm grip on the eye during wound construction and capsulorhexis. This is certainly a drawback during learning or teaching. Topical anaesthesia may not be so satisfactory for longer procedures, including management of complications, vitrectomy or conversion to extracapsular surgery.

Posterior sub-Tenon's delivery as described by Stevens $^{13}$ gives a rapid onset of anaesthesia with akinesia, as the agent used diffuses around the muscle sheaths and into the muscle cone. There is minimal effect on intraocular pressure ${ }^{14}$ and pressure-lowering devices are not required if volumes of $3-4 \mathrm{ml}$ are used. In addition there is often a degree of orbicularis block, and a separate lid block is seldom used, particularly with small-incision surgery. Prilocaine $2 \%$ is not an agent familiar to all ophthalmologists, but it has several advantages over more commonly used drugs. ${ }^{15}$ It is more diffusible and less irritant than lignocaine, and produces a block which lasts approximately twice as long. It is considerably less systemically toxic than bupivicaine. It may be possible, therefore, to achieve a speedy, long-lasting block without mixing with hyaluronidase.

The aim of this study was to compare prospectively these two techniques in routine cataract surgery; in particular their efficacy, patient tolerance, and unwanted effects. We also draw comparisons with previously published data on other techniques. 


\section{METHODS}

Fifty consecutive patients admitted for a routine day case list were asked for informed consent and randomised into two groups of 25 , to receive topical or sub-Tenon's local anaesthesia. Exclusion criteria were: previous intraocular surgery, inflammation or injury; a pupil less than $5 \mathrm{~mm}$ in diameter when fully dilated; and inability to understand a visual analogue pain scale. Both groups underwent identical preoperative preparation with g. phenylephrine $2.5 \%$, g. cyclopentolate $1 \%$ and g. diclofenac $0.1 \% 30$ minutes prior to surgery.

Sub-Tenon's local anaesthesia was administered by one surgeon (T.D.M.). Two drops of amethocaine $1 \%$ were applied to the cornea and conjunctiva and a small 'bleb' raised in the inferonasal quadrant $5 \mathrm{~mm}$ from the limbus with $0.1-0.2 \mathrm{ml} 2 \%$ prilocaine via a 25 gauge needle. A nick was made simultaneously through conjunctiva and Tenon's capsule with curved Westcott scissors and a purpose-designed subTenon's cannula was advanced to the equator. One millilitre of $2 \%$ prilocaine was injected and the cannula was slipped further round the globe to place another $1.5 \mathrm{ml}$ in the posterior sub-Tenon's space. No compressive device was used and surgery began after 5 minutes. At 5 minutes scores were given for motility and the extent of subconjunctival haemorrhage was noted. Motility was scored as: 0 , no movement/twitch only; 1 , movement in one direction of gaze; 2 , movement in two directions of gaze; 3 , movement in three or more directions with some restriction; 4, full eye movements. Subconjunctival haemorrhage was noted according to the number of quadrants affected. No 'top up' injections were made and surgery proceeded even if eye movements were unrestricted.

The topical group had three drops of $1 \%$ amethocaine instilled over a 10 minute period prior to surgery. At the start of surgery $0.1 \mathrm{ml} 2 \%$ lignocaine was injected subconjunctivally $2 \mathrm{~mm}$ posterior to the superior limbus to facilitate painless cautery of the scleral vessels.

All surgery was carried out by one surgeon (R.L.B.) and was standardised for each case. A 5 $\mathrm{mm}$ frown incision with $2-3 \mathrm{~mm}$ scleral pocket was made at 12 o'clock, followed by capsulorhexis and bimanual nucleofractis phacoemulsification. A $5.5 \mathrm{~mm}$ intraocular lens was inserted into the capsular bag and the wound left unsutured. The

Table I. Patient data

\begin{tabular}{lll}
\hline & Topical group & Sub-Tenon's group \\
\hline Number & 25 & 25 \\
Average age (years) & 75.7 & 78.9 \\
Males/females & $11 / 14$ & $5 / 20$ \\
Pupil size (mm) & 8.0 & 7.6 \\
Nuclear density & $2.6+$ & $2.8+$ \\
Operating time (min) & 14.4 & 14.8 \\
\hline
\end{tabular}

pupil size, duration of surgery, and any complications were noted. Nucleus density was judged on a scale of 1 to 5 according to ease of emulsification.

Patients were asked immediately post-operatively to score discomfort or pain on a visual analogue scale of 0 to 10 . All were asked in a standard format by one independent observer (day case nurse) in a separate room from other pre- and post-operative patients. The sub-Tenon's group were asked for pain scores for the local anaesthetic administration and for the surgery and the topical group were asked for a score regarding the surgery only.

\section{RESULTS}

Fifty patients were included in the trial, 25 in each group. Patient data are given in Table I. Three patients were excluded: one because the pupil was too small, one because of previous surgery and one because a deep-set eye required a temporal incision. Results are summarised in Table II.

In the topical group 13 of 25 subjects recorded a pain score of 1 or more, indicating that most felt something at least during the procedure. The difference between the pain scores in the two groups is highly statistically significant $(p<0.003$, MannWhitney $U$-test).

\section{DISCUSSION}

Both types of anaesthesia provided satisfactory operating conditions, and no complications were noted in any case. Operating time was similar, although it was noted by the surgeon that it is easier to rotate the eye downwards for wound construction, etc., in the topical group. This is partially aided by voluntary movement, but after the sub-Tenon's injection the medial and inferior recti are weakened preferentially, and any residual movement tends to be up and out. Some surgeons have expressed concern about the lack of akinesia during intraocular surgery with minimal anaesthesia, and some surgeons also favour a facial block. In our experience and that of others the eye can be safely controlled with an instrument such as a twist grip or appropriate forceps during wound construction and capsulorhexis and thereafter bimanual control is easy. The lack of a facial block is of little concern in nearly all cases with a self-sealing wound and formed eye throughout the operation. Of some concern are the significantly higher scores on the pain ranking associated with the

Table II. Pain, akinesia and subconjunctival haemorrhage scores

\begin{tabular}{|c|c|c|}
\hline & Topical group & Sub-Tenon's group \\
\hline Number & 25 & 25 \\
\hline Pain on injection & $\mathrm{n} / \mathrm{a}$ & 0 \\
\hline Pain on surgery & 0.46 (range $0-3$ ) & 0.02 (range $0-1)$ \\
\hline Conjunctival haemorrhage & $\mathrm{n} / \mathrm{a}$ & 1.5 (range $1-3$ ) \\
\hline Movement score & $\mathrm{n} / \mathrm{a}$ & 0.7 (range $0-2$ ) \\
\hline
\end{tabular}


topical group. More than half the patients felt something unpleasant during surgery, although only one case scored more than 1 on the analogue scale, and all were happy with their anaesthesia.

It should be noted that the topical group did receive a very small subconjunctival bleb of lignocaine at 12 o'clock. This was to allow painless cautery in the wound area. A clear corneal approach would obviate this (in our opinion) necessary additional procedure.

The sub-Tenon's group had impressive results as regards both the apparent painlessness of the administration of anaesthesic and the subsequent pain-free surgery. Heyworth and Seward ${ }^{2}$ using similar scoring found an average pain score of 2.6 on peribulbar injection, and other groups have reported similar results. ${ }^{1}$ Apart from its other advantages as regards the very low risk of ocular perforation, intravenous or intrathecal injection, subTenon's delivery seems to be very stress-free for the patient.

A significant number of patients did not achieve full akinesia, with most having significant movement in one direction of gaze at least. This evidently did not affect the effectiveness of the anaesthetic, and with the proviso made above that residual superior rectus activity can impede forced downgaze, it did not trouble the surgeon either. It is accepted that this surgeon is used to working with significant eye movement, but the block compares well with other more invasive methods. No top-up injections were given, and second doses are often given with other methods to achieve full akinesia. A significant drawback is the subconjunctival haemorrhage which occurs in one quadrant in every case and can spread to three. This does not impede either the effectiveness of the block or the surgery, but it is unsightly for the patient and may take several days to clear. In our experience, unlike others, ${ }^{13}$ the haemorrhage is not prevented by prior cautery or adrenaline drops, and often originates from episcleral vessels caught on introduction of the cannula. Modification to produce a more rounded atraumatic tip may be helpful.

Prilocaine has been largely overlooked as a local anaesthetic agent in ophthalmic surgery, but it may have contributed to the good results in this study. In particular the decreased local irritation compared with lignocaine may have made the administration more comfortable. The longer duration of block was not important in this study, but could be a significant advantage for difficult operating conditions, longer procedures such as detachment surgery, for teaching or for inexperienced surgeons.

The joint Royal Colleges report on local anaesthesia recommended the presence of an anaesthetist during local anaesthesia for cataract surgery. The complications directly attributable to the local anaesthesia are taken from large studies and case reports relating to either retrobulbar or peribulbar techniques. In the report it is agreed that minor procedures such as lid procedures under local anaesthesia would not require anaesthetist cover. Many of the complications unrelated to the local anaesthetic are simply the result of elderly, often unfit patients undergoing stressful procedures. ${ }^{16}$ Many units still find difficulty in establishing regular anaesthetic cover, and this is exacerbated in areas with either separate eye units or shortages of specialist anaesthetists. Representatives of both Colleges have agreed to review their advice in the light of new techniques.

We believe that both the techniques described in this study are safe, and individually or in combination can be used to cover all local anaesthesia requirements for intraocular surgery. Each has advantages and disadvantages, but both are significant advances over blind intraorbital injections with sharp needles. In particular, topical local anaesthesia is suited to shorter procedures, uncomplicated cases, experienced surgeons, and may require a degree of patient selection in view of the minor discomfort. In our view there is little place for sedation. Sub-Tenon's delivery is ideal for complicated cases, inexperienced surgeons, nervous patients, and is easily given during a procedure if topical anaesthesia becomes insufficient, for example after vitreous loss. It is suggested that provided further and wider experience proves the safety of these methods, the College guidelines may be modified.

Key words: Local anaesthesia, Cataract.

\section{REFERENCES}

1. Maclean H, Burton RL, Murray A. Randomised trial of patient comfort under topical versus peribulbar anaesthesia. J Cataract Refract Surg (under review).

2. Heyworth PLO, Seward HC. Local anaesthesia: the ophthalmologist's view. Eur J Implant Ref Surg 1993; 5:12-6.

3. Royal College of Anaesthetists, Royal College of Ophthalmologists. Report of the joint working party on anaesthesia in ophthalmic surgery, March 1993.

4. Nicoll JMV, Aryhara PA, Ahlem K, Baguneid S, Edge KR. Central nervous system complications after 6000 retrobulbar blocks. J Anaesth Analges 1987;66: 1298-302.

5. Brookshire GL, Gleitman KY, Schenk EC. Life threatening complications of retrobulbar block. Ophthalmology 1986;93:1476-8.

6. Rosenblatt RM, May DR, Barsoumian K. Cardiopulmonary arrest after retrobulbar block. Am J Ophthalmol 1980;90:425-7.

7. Kimble JA, Morris IE, Witherspoon CD, Feist RM. Globe perforation from peribulbar injection. Arch Ophthalmol 1987;105:479.

8. Hay A, Flynn HW, Hoffman JI, Reverra AH. Needle penetration of the globe during retrobulbar and peribulbar injection. Ophthalmology 1981;98:1017-24. 
9. Fichman RA. Topical eyedrops replace injection for anaesthesia. Ocular Surg News 1992;[March]:1.

10. Grabow HB. Topical anaesthesia for cataract surgery. Eur J Implant Ref Surg 1993;5:20-3.

11. Kershner RM. Topical anaesthesia for small incision self-sealing cataract surgery. J Cataract Refract Surg 1993;19:290-2.

12. Nielsen PJ. A prospective evaluation of anxiety and pain with topical analgesia or retrobulbar anaesthesia. Eur J Implant Ref Surg 1995;7:6-10.

13. Stevens JD. A new local anaesthesia technique for cataract extraction by one quadrant sub-Tenon's infiltration. Br J Ophthalmol 1992;76:670-4.

14. Stevens JD, Giubilei M, Lanigan L, Hykin P. SubTenon, retrobulbar and peribulbar local anaesthesia. Eur J Implant Ref Surg 1993;5:25-9.

15. Brahma AK, Pemberton CJ, Ayeko M, Morgan LH. Single medical injection peribulbar anaesthesia using prilocaine. Anaesthesia 1994;49:1003-5.

16. Rozanski A, et al. Mental stress and the induction of silent myocardial ischaemia. N Engl J Med 1988;318:21. 